\title{
Energy Spectrum of Bloch Electrons Under Checkerboard Field Modulations
}

\author{
Ming-Che Chang \\ Department of Physics, National Taiwan Normal University, Taipei, Taiwan \\ Min-Fong Yang \\ Department of Physics, Tunghai University, Taichung, Taiwan
}

(Dated: October 30, 2018)

\begin{abstract}
Two-dimensional Bloch electrons in a uniform magnetic field exhibit complex energy spectrum. When static electric and magnetic modulations with a checkerboard pattern are superimposed on the uniform magnetic field, more structures and symmetries of the spectra are found, due to the additional adjustable parameters from the modulations. We give a comprehensive report on these new symmetries. We have also found an electric-modulation induced energy gap, whose magnitude is independent of the strength of either the uniform or the modulated magnetic field. This study is applicable to experimentally accessible systems and is related to the investigations on frustrated antiferromagnetism.
\end{abstract}

PACS numbers: 72.20.My; 73.21.-b; quantum wells, mesoscopic, and nanoscale systems 73.43.-f; 75.10.Jm

\section{INTRODUCTION}

When the spectrum of a two-dimensional (2D) Bloch electron in a uniform magnetic field is plotted in the energy-flux diagram, a self-similar structure with fractal property emerges $\frac{1}{\underline{1}}$ Such a complex structure, called the Hofstadter spectrum, arises due to the commensurability between two length scales in this system: the lattice constant and the cyclotron radius. The Hofstadter spectrum is one of the earliest predictions of fractal structure in solid-state physics. Subsequently, it was found that not only the energy spectrum has self-similarity, the wave function also exhibits scaling behavior and can be analyzed using the renormalization group $\underline{\underline{*}}$

Because of its beautiful structure, the Hofstadter spectrum has attracted many researchers' attention, and the spectra for different 2D lattice symmetries have been reported. Besides the square lattice, there are also triangular lattice,,$\frac{3}{=}$ honeycomb lattice, $\stackrel{4}{=}$ Kagome lattice,,$\frac{5}{5}$ and a bipartite periodic structure with hexagonal symmetry $\underline{6}$ These are all studied within the framework of Bloch electrons in a uniform magnetic field, usually assuming nearest-neighbor $(\mathrm{NN})$ couplings $t_{1}$ only. Including and varying the next-nearest-neighbor (NNN) couplings $t_{2}$ leads to band-crossings accompanied by exchange of quantized Hall conductances between bands $\underset{7.8}{ }$ For a square lattice, detailed scaling analysis reveals a bicritical point at $t_{1}=2 t_{2}$, accompanied by interesting topological change of the spectrum $\frac{9}{9}$ Spectra for systems with couplings beyond next-nearest neighbors have also been studied $\stackrel{10}{=}$ In addition, the external magnetic field, rather than being uniform, can be periodically modulated with a pattern unrelated to the original lattice. The simplest situation when a magnetic lattice overlaps with the electric lattice is realized when a ferromagnetic grid is deposited on a semiconductor ${ }^{11}$ The interfacial stress between two materials would naturally induce an electric grid with the same period and symmetry as the ferromagnetic grid. More generally, there can also be a magnetic modulation with the pattern of a $1 \mathrm{D}$ strip, $\frac{12}{2}$ or a $2 \mathrm{D}$ checkerboard ${ }^{13.14 .15}$ superimposes on the electric square lattice. The checkerboard configuration has been realized experimentally using a superconducting Nb-network with periodic magnetic Dy-islands $\frac{15}{n}$ The calculations of the Hofstadter spectra provide the basis to study such articifial networks.

A direct observation of the Hofstadter spectrum has been realized using microwaves 16 or acoustic waves 17 transmitting through an array of macroscopic scatters. However, a fractal electronic spectrum is significantly more difficult to be realized in an usual solid, whose lattice constant is only a few angstroms, and a magnetic field of the order of $10^{4}$ Teslas is required. For 10 Teslas or less, we can only probe the part of the Hofstadter spectrum that reduces to the familiar Landau levels with roughly equal spacings in energy. In the last decade, different superlattice structures with much larger lattice constants are used to cope with this high-field problem. 18 Besides, several physical systems are closely related to the Hofstadter problems and offer alternative angles of investigation, for example, the studies of a superconductor in a vortex state, 19 a superconducting network in a magnetic field $\stackrel{15}{15}$ and a junction of three quantum wires $\stackrel{20}{2}$ Furthermore, recent advance on optical lattices makes it possible to implement a lattice Hamiltonian resembling the effects of magnetic fields with neutral atoms ${ }^{21}$ This offers great opportunities since not only the magnetic field, but also the lattice symmetry, the potential strength, and the relative importance of many-body effects can be adjusted in such a system.

This paper is motivated by a study very different from those mentioned above. In a recent paper, ${ }^{22}$ Misguich and coworkers, by using the hard-core bosons to represent the spin degrees of freedom, and using the Chern-Simons transformation to transmute bosons to fermions, mapped a 2D frustrated antiferromagnetic problem to a Hofstadter problem. This approach is subsequently used to study the magnetization properties of the $J_{1}-J_{2}$ Heisen- 
berg model on a square lattice. ${ }^{23}$ After suitable mathematical mappings and a mean-field approximation, the magnetization problem can be reduced to a Hofstadter problem with both electric and magnetic super-structures superimpose on the original lattice. This motivates us to consider the checkerboard super-structure, which is related to the Néel phase in the magnetization problem, with congruous electric and magnetic modulations. Couplings up to next nearest-neighbors are considered, which are essential to cause magnetic frustration in the $J_{1}-J_{2}$ Heisenberg model. ${ }^{24}$

In this paper, we make a comprehensive survey of the symmetries of the Hofstadter spectra with field modulations. Some of the symmetries already exist without modulations, such as the ones related to reversing the direction of the magnetic flux, and shifting the flux in a plaquette by two flux quanta (see items II and III in Sec. II). Some of the other symmetries that are closely related to field modulations are reported for the first time. In particular, when the system is subject to staggered $\pi$-fluxes, its spectrum in the $E-\phi$ diagram has an updowm symmetry even with NNN couplings (see Fig. 5), which is quite unexpected since NNN couplings usually destroy such a symmetry ${ }^{7}$ Besides the studies on symmetries, for systems with only NN couplings, we find a simple algebraic connection between the spectra with and without the electric checkerboard field. We also find a flux-independent energy gap induced by the electric modulation (see Fig. 3), which can be explained by using the algebraic relation just mentioned.

This paper is organized as follows. Theoretical formulation on the system with checkerboard super-structure, as well as the discussion of the symmetries of the energy spectra, can be found in Sec. II. Major features of the Hofstadter spectra are discussed in Sec. III. We summarize and conclude our results in Sec. IV. The proofs on checkerboard-translation symmetry of the spectrum and on the existence of the flux-independent energy gap are given in the appendices.

\section{THEORETICAL ANALYSIS}

The tight-binding Hamiltonian describing the motion of an electron in a magnetic field is given by

$$
H=-\frac{1}{2} \sum_{\langle i, j\rangle}\left(t_{i j} e^{i \theta_{i j}} f_{i}^{+} f_{j}+\text { h.c. }\right)+\sum_{i} V_{i} f_{i}^{+} f_{i},
$$

where $\theta_{i j}$ is the magnetic phase factor. For clarification, we will replace the label $i$ by $(n, m)$ in the following, which denotes the $(n, m)$ plaquette as well as the lattice point at the lower left corner of the plaquette. Without loss of generality, we take the uniform part of the magnetic flux through plaquette as $\phi=2 \pi p / q$ with relative prime integers $p$ and $q$. For the checkerboard modulation, we have $\delta \phi_{i} / 2 \pi=-\Delta_{\phi}(-1)^{n+m}$ and $V_{i} \equiv \Delta_{V}(-1)^{n+m}$ (see Fig. 1). The Landau gauge is used such that the magnetic phase factors become

$$
\left\{\begin{array}{l}
\theta_{n+1, m ; n, m}=0, \\
\theta_{n, m+1 ; n, m}=n \phi+(-1)^{n+m} \pi \Delta_{\phi}, \\
\theta_{n+1, m+1 ; n, m}=\theta_{n, m+1 ; n+1, m}=\left(n+\frac{1}{2}\right) \phi .
\end{array}\right.
$$

Due to the modulation in the $y$ direction and under the gauge choice in Eq. (2), the tight-binding Hamiltonian in Eq. (1) now becomes invariant under the $y$-translation $m \rightarrow m+2$. Thus the Bloch theorem gives

$$
f_{n, m}=e^{-i k_{y} m} c_{n, m}\left(k_{y}\right)
$$

for $\left|k_{y}\right| \leq \pi / 2$, where $c_{n, m+2}\left(k_{y}\right)=c_{n, m}\left(k_{y}\right)$ and $c_{n, m}\left(k_{y}+\pi\right)=c_{n, m}\left(k_{y}\right)$. Therefore, the generalized Harper equation becomes

$$
\begin{aligned}
& \mathbf{A}_{n} \vec{c}_{n}\left(k_{y}\right)+\mathbf{B}_{n} \vec{c}_{n+1}\left(k_{y}\right)+\mathbf{B}_{n-1} \vec{c}_{n-1}\left(k_{y}\right) \\
& =E \vec{c}_{n}\left(k_{y}\right)
\end{aligned}
$$

where $\vec{c}_{n}\left(k_{y}\right)=\left(c_{n, 1}\left(k_{y}\right), c_{n, 2}\left(k_{y}\right)\right)^{T}$ and

$$
\begin{gathered}
\mathbf{A}_{n}=\left(\begin{array}{cc}
-(-1)^{n} \Delta_{V} & -t_{1} \cos \left(\chi_{n}\right) e^{i \delta_{n}} \\
-t_{1} \cos \left(\chi_{n}\right) e^{-i \delta_{n}} & (-1)^{n} \Delta_{V}
\end{array}\right) \\
\mathbf{B}_{n}=\left(\begin{array}{cc}
-t_{1} / 2 & -t_{2} \cos \left(\eta_{n}\right) \\
-t_{2} \cos \left(\eta_{n}\right) & -t_{1} / 2
\end{array}\right)
\end{gathered}
$$

with $\chi_{n}=n \phi+k_{y}, \delta_{n}=(-1)^{n} \pi \Delta_{\phi}$, and $\eta_{n}=(n+$ $1 / 2) \phi+k_{y}$. It can be easily checked that

$$
\mathbf{A}_{n+Q}=\mathbf{A}_{n}, \mathbf{B}_{n+Q}=\mathbf{B}_{n}
$$

where $Q=q(2 q)$ for an even (odd) integer $q$. Thus, the $n$ in Eq. (4) satisfies the condition $1 \leq n \leq Q$. Besides, because of the magnetic translation symmetry, the primitive unit cell is consisted of $q(Q)$ plaquettes without (with) checkerboard modulation.

The Bloch condition along the $x$ direction can be written as

$$
c_{n, m}\left(k_{y}\right)=e^{-i k_{x} n} \psi_{n, m}\left(k_{x}, k_{y}\right)
$$

for $\left|k_{x}\right| \leq \pi / Q$, where $\psi_{n+Q, m}\left(k_{x}, k_{y}\right)=\psi_{n, m}\left(k_{x}, k_{y}\right)$ and $\psi_{n, m}\left(k_{x}+2 \pi / Q, k_{y}+\pi\right)=\psi_{n, m}\left(k_{x}, k_{y}\right)$. Now we only need to solve the problem within the first magnetic Brillouin zone given by $\left|k_{x}\right| \leq \pi / Q$ and $\left|k_{y}\right| \leq \pi / 2$.

Thus we obtain the eigenvalue equation, $\mathbf{M} \Psi=E \Psi$, where $\Psi=\left(\psi_{1,1}, \psi_{1,2}, \psi_{2,1}, \psi_{2,2}, \cdots, \psi_{Q, 1}, \psi_{Q, 2}\right)^{T}$ and 


$$
\mathbf{M}=\left(\begin{array}{ccccccc}
\mathbf{A}_{1} & \mathbf{B}_{1} e^{-i k_{x}} & 0 & \cdots & 0 & 0 & \mathbf{B}_{Q} e^{i k_{x}} \\
\mathbf{B}_{1} e^{i k_{x}} & \mathbf{A}_{2} & \mathbf{B}_{2} e^{-i k_{x}} & \cdots & 0 & 0 & 0 \\
0 & \mathbf{B}_{2} e^{i k_{x}} & \mathbf{A}_{3} & \cdots & 0 & 0 & 0 \\
\cdots & \cdots & \cdots & \cdots & \cdots & \cdots & \cdots \\
0 & 0 & 0 & \cdots & \mathbf{B}_{Q-2} e^{i k_{x}} & \mathbf{A}_{Q-1} & \mathbf{B}_{Q-1} e^{-i k_{x}} \\
\mathbf{B}_{Q} e^{-i k_{x}} & 0 & 0 & \cdots & 0 & \mathbf{B}_{Q-1} e^{i k_{x}} & \mathbf{A}_{Q}
\end{array}\right)
$$

We calculate the energy eigenvalues for all the values of $\vec{k}$ in the first magnetic Brillouin zone, $\left|k_{x}\right| \leq \pi / Q$ and $\left|k_{y}\right| \leq \pi / 2$, by directly diagonalizing the $2 Q \times 2 Q$ Hamiltonian matrix $\mathbf{M}(\vec{k})$. As indicated in Fig. 1, the system has the checkerborad translational symmetry. That is, the system is invariant under the lattice translation by two lattice constants along either the $x$ or the $y$ directions, or under the translation $(n, m) \rightarrow(n+1, m+1)$ along the diagonal. Thus one expects that, under the above transformations, the energy spectrum obtained by the eigenvalue problem with the Hamiltonian matrix $\mathbf{M}(\vec{k})$ should remain the same, which is not obvious as seen from Eq. (9). In Appendix A, we prove that the Hamiltonian matrices before and after the translations are identical up to a shift in $k_{y}$ and thus give the same energy spectra.

We show that there are several general symmetries of the spectra in the $E-\phi$ diagram, which can be used to reduce the amount of calculations. Similar discussion for the systems without field modulations can be found in Ref. [7]. In the following, the collecton of energy subbands at a flux $\phi$ (per plaquette) modulated by $\left(\Delta_{\phi}, \Delta_{V}\right)$ is denoted by $E\left(\phi, \Delta_{\phi}, \Delta_{V}\right)$. It has the following symmetries: ${ }^{25}$

I. $E\left(\phi, \Delta_{\phi}, \Delta_{V}\right)=E\left(-\phi,-\Delta_{\phi}, \Delta_{V}\right)$

This follows from using two (three-dimensional) coordinate systems which are mirror images of each other with respect to the $x-y$ plane. The physics, and hence the energy spectra, should be the same in these two frames with opposite handnesses.

II. $E\left(\phi, \Delta_{\phi}, \Delta_{V}\right)=E\left(-\phi, \Delta_{\phi}, \Delta_{V}\right)=$ $E\left(-\phi,-\Delta_{\phi},-\Delta_{V}\right)$

The first equality follows from rotating the (threedimensional) coordinate frame by 180 degrees around either the $x$-axis or the $y$-axis; the second is from shifting the coordinate by one plaquette along either the $x$-axis or the $y$-axis.

III. $E\left(\phi, \Delta_{\phi}, \Delta_{V}\right)=E\left(\phi+2 \phi_{0}, \Delta_{\phi}, \Delta_{V}\right)$

This results from the following two facts: (i) the smallest hopping loop for electrons encloses half of a plaquette; (ii) the Aharonov-Bohm phase for this closed loop is unchanged after adding one flux quantum to within this loop.

IV. $E\left(\phi, \Delta_{\phi}, \Delta_{V}\right)=\Re E\left(\phi+\phi_{0},-\Delta_{\phi}, \Delta_{V}\right)$, where the operator $\Re$ flips the spectrum with respect to the horizontal $E=0$ line.

This follows from the two transformations: (i) $f_{n, m} \rightarrow(-1)^{n+m} f_{n, m}$ and $\phi \rightarrow \phi+\phi_{0}$; (ii) $f_{n, m} \rightarrow$ $f_{n, m+1}$ and $\Delta_{\phi} \rightarrow-\Delta_{\phi}$. It can be shown that the overall sign of the Hamiltonian in Eq. (11) changes after this two transformations, thus the spectra should have symmetry IV after shifting $\phi$ by one $\phi_{0}$ and reversing the direction of $\Delta_{\phi}$.

V. $E\left(\phi, \Delta_{\phi}, \Delta_{V}\right)=E\left(\phi-\phi_{0}, \Delta_{\phi}-1, \Delta_{V}\right)$

This is because of the freedom in shifting the fluxes, $\left(\phi_{A}, \phi_{B}\right) \rightarrow\left(\phi_{A}-2 \phi_{0}, \phi_{B}\right)$, where $\phi_{A}=\phi+2 \pi \Delta_{\phi}$ and $\phi_{B}=\phi-2 \pi \Delta_{\phi}$ are the fluxes through each plaquette of the $A$ and $B$ sublattices respectively.

By combining symmetries I and II, it is not difficult to see that $E\left(\phi, \Delta_{\phi}, \Delta_{V}\right)$ should remain unchanged when the sign of any of its arguments, $\phi, \Delta_{\phi}$, or $\Delta_{V}$, is changed. From symmetries III and II, we have $E\left(\phi_{0}+\right.$ $\left.\phi, \Delta_{\phi}, \Delta_{V}\right)=E\left(\phi-\phi_{0}, \Delta_{\phi}, \Delta_{V}\right)=E\left(\phi_{0}-\phi, \Delta_{\phi}, \Delta_{V}\right)$. That is, the distribution of $E(\phi)$ in the $E-\phi$ diagram has a mirror symmetry with respect to the vertical line $\phi=\phi_{0}$.

Finally, we show that, in the $E-\phi$ diagram, it is sufficient to plot the spectra within the range $0 \leq \phi<\phi_{0} / 2$ only. The reason is as follows: from II, III, IV, and the freedom to flip the signs of $\Delta_{\phi}$ and $\Delta_{V}$ without changing the spectrum, it is clear that, for fixed values of $\Delta_{\phi}$ and $\Delta_{V}$, it suffices knowing the spectrum within the interval $\left[0, \phi_{0}\right)$. Moreover, from IV and I, we have $E\left(\phi_{0} / 2+\phi, \Delta_{\phi}, \Delta_{V}\right)=\Re E\left(\phi-\phi_{0} / 2,-\Delta_{\phi}, \Delta_{V}\right)=$ $\Re E\left(\phi_{0} / 2-\phi, \Delta_{\phi}, \Delta_{V}\right)$. Therefore, the spectrum along the whole flux-coordinate can be determined by the $E(\phi)$ within the interval $\left[0, \phi_{0} / 2\right)$.

\section{MAIN FEATURES OF THE SPECTRA}

In the discussion below, all energies are in units of $t_{1}$. Besides $t_{1}(=1)$, there are three adjustable parameters in the present generalized Hofstadter model on a square lattice: $\Delta_{\phi}, \Delta_{V}$, and $t_{2}$. It is impossible to show all the results from the whole three-dimensional parameter space $^{26}$ Therefore, we selectively report on certain sets of parameters with representative features. Notice that in Refs. 13 14 15], neither electric modulation nor NNN hoppings has been considered and the parameter space is one dimensional only. 
First, we consider the effect of the checkerboard modulation on the systems without NNN couplings $t_{2}$, which have several symmetries in addition to the symmetries $\mathrm{I} \sim \mathrm{V}$ listed above.

III. $E\left(\phi, \Delta_{\phi}, \Delta_{V}\right)=E\left(\phi+\phi_{0}, \Delta_{\phi}, \Delta_{V}\right)$

When there is only NN hoppings, the period of the spectrum is one flux quantum since the smallest loop of hopping now is one plaquette, instead of half of the plaquette.

IV. $E\left(\phi, \Delta_{\phi}, \Delta_{V}\right)=\Re E\left(\phi, \Delta_{\phi}, \Delta_{V}\right)$

This results from symmetries IV and III', followed by flipping the sign of $\Delta_{\phi}$, which would not change the spectrum. Thus, $E\left(\phi, \Delta_{\phi}, \Delta_{V}\right)$ is symmetric with respect to the horizontal $E=0$ line when $t_{2}$ $=0$.

ㅍ. $E\left(\phi, \Delta_{\phi}, \Delta_{V}\right)=E\left(\phi-\phi_{0} / 2, \Delta_{\phi}-1 / 2, \Delta_{V}\right)$

The argument is similar to the one leading to $\mathrm{V}$, except that now $\phi_{A}$ can be shifted by one $\phi_{0}$ without altering the Aharonov-Bohm phase of a closed-loop hopping.

In Fig. 2, the spectrum for a checkerboard modulation with $\left(\Delta_{\phi}, \Delta_{V}\right)=(0.1,0)$ is presented. The spectrum is indeed symmetric with respect to the $E=0$ line, according to symmetry IV'. Furthermore, because of the symmetry $\mathrm{V}^{\prime}$ and the freedom to flip the signs of the arguments, we have $E\left(\phi_{0} / 4+\phi, \Delta_{\phi}, \Delta_{V}\right)=E\left(\phi-\phi_{0} / 4,1 / 2-\right.$ $\left.\Delta_{\phi}, \Delta_{V}\right)=E\left(\phi_{0} / 4-\phi, \Delta_{\phi}-1 / 2, \Delta_{V}\right)$. Therefore, after being reflected by the vertical line at $\phi_{0} / 4$, Fig. 2 with $\Delta_{\phi}=0.1$ is identical to the Hofstadter spectrum for $\Delta_{\phi}=0.427$

In Fig. 3, a checkerboard modulation with $\left(\Delta_{\phi}, \Delta_{V}\right)=$ $(0.1,0.1)$ is considered. Without NNN hoppings, this Hofstadter spectrum retains the same symmetries as in Fig. 2. However, a distinctive $\phi$-independent energy gap with a magnitude $E_{g}=2 \Delta_{V}$ appears in the middle [also see Fig. 6(a)]. This is true with or without adding the modulation $\Delta_{\phi}$. First, it is not difficult to understand why the spectrum splits to two groups in energy: they originate from the two Bloch bands at $\phi=0$ due to the checkerboard modulation of the scalar potential. What is surprising is that the magnitude of the gap remains a constant for different $\phi$ 's and $\Delta_{\phi}$ 's. It is no longer a constant as long as NNN couplings are included (see Fig. 4). A proof of the existence of the flux-independent gap is given in Appendix B, where it is shown that there exists a very simple relation between the spectra with and without electrostatic modulation $\Delta_{V}$. That is, $E\left(\phi, \Delta_{\phi}, \Delta_{V}\right)= \pm\left[E\left(\phi, \Delta_{\phi}, 0\right)^{2}+\Delta_{V}^{2}\right]^{1 / 2}$. It can be checked that the spectra in Fig. 2 and Fig. 3 do obey this relation in details.

The constancy of the energy gap in the limit of small flux $\phi$ can be understood in the following semiclassical picture ${ }^{28}$ The energy bands with vanishing widths as $\phi \rightarrow 0$ in Fig. 3 are the cyclotron energy levels of the two parent bands at $\phi=0$, which have the energy dispersions
$E_{ \pm}(\vec{k})= \pm\left[\left(\cos k_{x}+\cos k_{y}\right)^{2}+\Delta_{V}^{2}\right]^{1 / 2}$ if $\Delta_{\phi}=0$. It can be shown that, near the two inner band edges with energies $E_{ \pm}= \pm \Delta_{V}$, the cyclotron effective masses approach infinity. Therefore, the position of the lowest Landau level approaches the lowest possible energy at the band edge and does not depend on the uniform magnetic field.

When NNN couplings are included, the spectrum immediately lose the mirror symmetry with respect to the horizontal $E=0$ line ${ }^{7}$ If only two of the three parameters are nonzero, then the spectrum remains fractal but distorted. When all three parameters, $\Delta_{\phi}, \Delta_{V}$, and $t_{2}$, are nonzero, the subbands become significantly wider in most, but not all, of the regions. A typical example is shown in Fig. 4. The extent of widening varies as the parameters are varied. Because of the widening, the electrons are more delocalized, and become more mobile in transport.

There is a surprising exception to the asymmetry resulted from NNN hoppings: the symmetry is restored again when $\Delta_{\phi}=0.5$, even if both $\Delta_{V}$ and $t_{2}$ are nonzero. For example, the symmetric spectrum shown in Fig. 5 is for $\left(\Delta_{\phi}, \Delta_{V}, t_{2}\right)=(0.5,0.3,0.7)$. The existence of such a symmetry can be proved as follows. From the symmetries III, IV, and V listed above, and the freedom to flip the signs of the arguments, it can be shown that $E\left(\phi, \Delta_{\phi}, \Delta_{V}\right)=\Re E\left(\phi, 1-\Delta_{\phi}, \Delta_{V}\right)$, which is a far less apparent symmetry since it relates two systems with different strengths of flux modulation. It is clear that when $\Delta_{\phi}=0.5$, the spectrum has to be symmetric with respect to the line $E=0$.

In Fig. 6, we demonstrate how the continuous variation of $\Delta_{V}$ and $\Delta_{\phi}$ influence the spectrum. The uniform flux and the NNN couplings are fixed at the values of $p / q=2 / 5$ and $t_{2}=0$. In principle, there should be $Q=2 q=10$ bands at this value of the flux. However, in Fig. 6(a) with $\left(\Delta_{V}, \Delta_{\phi}\right)=(x, 0)$, where $x \in[0,1]$ is the value of the $x$-coordinate, only 6 bands are observed. In fact, each of the upper two and lower two bands is itself formed by two overlapping subbands. We can also see that the band gap in the middle is indeed proportional to $\Delta_{V}$, as mentioned earlier. In Fig. 6(b), $\left(\Delta_{V}, \Delta_{\phi}\right)=(0, x)$, where $x \in[0,1]$ is again the value of the $x$-coordinate. There is almost no similarity between (a) and (b). The overlapped subbands in Fig. 6(a) are split by a nonzero $\Delta_{\phi}$ and become very thin in most of the regions. On the other hand, the band in the middle is thick and is actually composed of two subbands. The increase of flux modulation also induces many band crossings. In addition, there is an apparent symmetry $E\left(\phi, \Delta_{\phi}, \Delta_{V}\right)=E\left(\phi, 1-\Delta_{\phi}, \Delta_{V}\right)$. In Fig. 6(c), both $\Delta_{V}$ and $\Delta_{\phi}$ are nonzero and have the same numerical value. It has mixing features from (a) and (b), but the magnitude of the energy gap in the middle is not altered [comparing with (a)] by the nonzero $\Delta_{\phi}$. Such a continuous tuning of the band structure might be realized in the future using the optical lattices formed by quantum optical means ${ }^{21}$ 


\section{SUMMARY}

The studies of Hofstadter spectrum have evolved from pure academic curiosities to accessible experimental investigations. It is a basic physics problem involving simple interplay between a lattice and a magnetic field. Because of its general setting, it is not surprising to find counterpart problems in different physical systems, such as the quantum Hall system, the type-II superconductivity, and the two-dimensional antiferromagnetism. Motivated by a study on the frustrated antiferromagnetism, and the recent experimental advances, we study the Hofstadter problem with checkerboard modulations in details. In this paper, the spectra are found to have several flux-related symmetries with respect to the change of $\phi$ and $\Delta_{\phi}$. One unanticipated symmetry occurs when $\Delta_{\phi}=1 / 2$. At that value, the spectrum are symmetric with respect to the $E=0$ line even in the presence of NNN hoppings. In the absence of NNN hoppings, we find a flux-independent energy gap induced by electric modulations. Furthermore, a simple connection between the spectra for bipartite systems with and without electric modulation is discovered. More detailed aspects of the spectra are not investigated in this paper, however, such as the change of the fractal measures in the $\Delta_{\phi}-\Delta_{V}-t_{2}$ parameter space. Such a study would reveal different phases in this space, as was done by Han and coworkers on the systems in a uniform magnetic field 9 The most general problem, when the superlattices of modulation can have the symmetries of their own, is considerally more involved. This study offers a starting point for researches in this direction.

\section{Acknowledgments}

M.C.C. and M.F.Y. acknowledge the financial support from the National Science Council of Taiwan under Contract Nos. NSC 91-2112-M-003-019 and NSC 91-2112-M029-007 respectively.

\section{APPENDIX A: PROOF OF THE CHECKERBOARD-TRANSLATION SYMMETRY OF THE SPECTRUM}

In this appendix, we show that the energy spectrum obtained from Eq. (9) does respect the checkerboard translation symmetry.

First, because there is no $m$-dependence of the matrix elements in Eq. (9), the Hamiltonian matrix and therefore the spectrum are unchanged under the lattice translation $m \rightarrow m+2$ such that $\psi_{n, m}\left(k_{x}, k_{y}\right) \rightarrow$ $\psi_{n, m+2}\left(k_{x}, k_{y}\right)$. Second, from Eqs. (5) and (6), one can show that the matrix elements in the Hamiltonian matrix satisfy the relations $\mathbf{A}_{n+2}\left(k_{y}\right)=\mathbf{A}_{n}\left(k_{y}+2 \phi\right)$ and $\mathbf{B}_{n+2}\left(k_{y}\right)=\mathbf{B}_{n}\left(k_{y}+2 \phi\right)$. Therefore, under the lattice translation $n \rightarrow n+2$ such that $\psi_{n, m}\left(k_{x}, k_{y}\right) \rightarrow$ $\psi_{n+2, m}\left(k_{x}, k_{y}\right)$, the new Hamiltonian matrix for the eigenvalue problem after transformation becomes identi- cal to the original one with another value of $k_{y}$, i.e., $k_{y} \rightarrow$ $k_{y}+2 \phi$. Thus the whole energy spectrum within the first magnetic Brillouin zone remains the same. Third, the matrix elements in the Hamiltonian matrix can be shown to obey the following identities: $\sigma_{x} \mathbf{A}_{n+1}\left(k_{y}\right) \sigma_{x}=$ $\mathbf{A}_{n}\left(k_{y}+\phi\right)$ and $\sigma_{x} \mathbf{B}_{n+1}\left(k_{y}\right) \sigma_{x}=\mathbf{B}_{n}\left(k_{y}+\phi\right)$, where $\sigma_{x}$ is the Pauli matrix. By using these identities, one can prove that, under the lattice translation $(n, m) \rightarrow(n+$ $1, m+1)$ such that $\psi_{n, m}\left(k_{x}, k_{y}\right) \rightarrow \psi_{n+1, m+1}\left(k_{x}, k_{y}\right)$, the new Hamiltonian matrix again becomes identical to the original one with a shift $k_{y} \rightarrow k_{y}+\phi$. Hence we conclude that the energy spectrum is indeed invariant under the checkerborad translation.

\section{APPENDIX B: PROOF OF THE EXISTENCE OF THE FLUX-INDEPENDENT ENERGY GAP}

For $t_{2}=0$, our model is a nearest-neighbor-hopping model on a bipartite lattice. Therefore, we can rewrite the Hamiltonian in Eq. (11) as

$$
H=\left(\left\{f_{A}^{\dagger}\right\},\left\{f_{B}^{\dagger}\right\}\right)\left(\begin{array}{cc}
\Delta_{V} I & \mathcal{D} \\
\mathcal{D}^{\dagger} & -\Delta_{V} I
\end{array}\right)\left(\begin{array}{l}
\left\{f_{A}\right\} \\
\left\{f_{B}\right\}
\end{array}\right),
$$

where $I$ denotes the identity matrix, $\left\{f_{A}\right\}=\left\{f_{n, m} \mid n+\right.$ $m$ is even $\}$ is a set of fermion operators for sublattice $A$ and $\left\{f_{B}\right\}=\left\{f_{n, m} \mid n+m\right.$ is odd $\}$ is for sublattice $B$. When $\Delta_{V}=0$, the Schrödinger equation is

$$
\left(\begin{array}{cc}
0 & \mathcal{D} \\
\mathcal{D}^{\dagger} & 0
\end{array}\right)\left(\begin{array}{l}
\Phi_{A} \\
\Phi_{B}
\end{array}\right)=E_{0}\left(\begin{array}{c}
\Phi_{A} \\
\Phi_{B}
\end{array}\right)
$$

where $E_{0}$ is the eigenvalue for the system with $\Delta_{V}=0$, and $\left(\Phi_{A}, \Phi_{B}\right)^{T}$ is the corresponding eigenvector. From them we can construct the eigenstates for the original problem:

$$
\begin{gathered}
\Phi_{+} \equiv\left(\begin{array}{c}
\Delta_{V}+\sqrt{E_{0}^{2}+\Delta_{V}^{2}} \Phi_{A} \\
\mathcal{D}^{\dagger} \Phi_{A}
\end{array}\right) \\
\Phi_{-} \equiv\left(\begin{array}{c}
\mathcal{D} \Phi_{B} \\
-\Delta_{V}-\sqrt{E_{0}^{2}+\Delta_{V}^{2}} \Phi_{B}
\end{array}\right)
\end{gathered}
$$

with the corresponding eigenvalues $E_{ \pm}= \pm \sqrt{E_{0}^{2}+\Delta_{V}^{2}}$, because

$$
\left(\begin{array}{cc}
\Delta_{V} I & \mathcal{D} \\
\mathcal{D}^{\dagger} & -\Delta_{V} I
\end{array}\right) \Phi_{ \pm}= \pm \sqrt{E_{0}^{2}+\Delta_{V}^{2}} \Phi_{ \pm} .
$$

Therefore, the energy spectrum is symmetric with respect to the horizontal $E=0$ line as mentioned in Sec. III. The positive-energy and the negative-energy parts are separated by an energy gap $2 \sqrt{\left|E_{0}\right|_{\text {min }}^{2}+\Delta_{V}^{2}}$, where $\left|E_{0}\right|_{\text {min }}$ is the minimum value of $\left|E_{0}\right|$ at given $\phi$ and $\Delta_{\phi}$. Since it has been shown that zero-energy modes exist in the absence of $\Delta_{V}$ for all flux values, ${ }^{29}$ we have $\left|E_{0}\right|_{\min }=0$ for all values of $\phi$ and $\Delta_{\phi}$. Consequently, the magnitude of the energy gap in the presence of $\Delta_{V}$ should be $2 \Delta_{V}$, independent of the values of $\phi$ and $\Delta_{\phi}$. 
1 D. R. Hofstadter, Phys. Rev. 14, 2239 (1976).

2 D. J. Thouless and Q. Niu, J. Phys. A, Math. Gen. 16, 1911 (1983); D. Dominguez, C. Wiecko, and J. Jose, Phys. Rev. B 45, 13919 (1992).

3 F. H. Claro and G. H. Wannier, Phys. Rev. B 19, 6068 (1979).

4 R. Rammal, J. Phys. (Paris) 46, 1345 (1985).

5 Y. Xiao, V. Pelletier, P. M. Chaikin, and D. A. Huse, Phys. Rev. B 67, 104505 (2003).

6 J. Vidal, R. Mosseri, and B. Doucot, Phys. Rev. Lett. 81, 5888 (1998).

7 Y. Hatsugai and M. Kohomoto, Phys. Rev. B 42, 4282 (1990).

8 M. Y. Lee, M. C. Chang, and T. M. Hong, Phys. Rev. B 57, 11895 (1998).

9 J. H. Han, D. J. Thouless, H. Hiramoto, and M. Kohmoto, Phys. Rev. B 50, 11365 (1994).

10 A. Barelli and R. Fleckinger, Phys. Rev. B 46, 11559 (1992).

11 P. D. Ye et al, Appl. Phys. Lett. 67, 1441 (1995); R. R. Gerhardts, D. Pfannkuche, and V. Gudmundsson, Phys. Rev. B 53, 9591 (1996).

12 G. Y. Oh, J. Jang, and M. H. Lee, J. Korean Phys. Soc. 28, 79 (1995); G. Y. Oh and M. H. Lee, Phys. Rev. B 53, 1225 (1996); P. Fekete and G. Gumbs, J. Phys.: Condens. Matter 11, 5475 (1999).

13 Q. W. Shi and K. Y. Szeto, Phys. Rev. B 56, 9251 (1997).

14 G. Y. Oh, Phys. Rev. B 60, 1939 (1999).

15 S. Ito, M. Ando, S. Katsumoto, and Y. Iye, J. Phys. Soc. Japan 68, 3158 (1999); M. Ando, S. Ito, S. Katsumoto, and Y. Iye, ibid 68, 3462 (1999).

16 U. Kuhl and H. J. Stockmann, Phys. Rev. Lett. 80, 3232 (1998).

17 O. Richoux and V. Pagneux, Europhys. Lett. 59, 34 (2002).

18 C. Albrecht, J. H. Smet, K. von Klitzing, D. Weiss, V. Umansky, and H. Schweizer, Phys. Rev. Lett. 86, 147 (2001).

19 Y. Morita and Y. Hatsugai, Phys. Rev. Lett. 86, 151 (2001); H. K. Nguyen and S. Chakravarty, Phys. Rev. B 65, 180519 (2002).

${ }^{20}$ C. Chamon, M. Oshikawa, and I. Affleck, cond-mat/0305121

21 D. Jaksch and P. Zoller, New J. Phys. 5, 56 (2003).

22 G. Misguich, Th. Jolicoeur, and S. M. Girvin, Phys. Rev. Lett. 87, 097203 (2001).

23 M. C. Chang and M. F. Yang, Phys. Rev. B 66, 184416 (2002).

24 The antiferromagnetic couplings $J_{1}$ and $J_{2}$ in the Heisenberg model are equal to $-t_{1}$ and $-t_{2}$ respectively. The nonzero $J_{2}$ coupling results in a frustrated antiferromagnetic system. After a mean-field approximation, it can be shown that $\Delta_{V}=4\left(J_{1}-J_{2}\right) \Delta_{\phi}$ (see Sec. II for the definitions of $\Delta_{\phi}$ and $\left.\Delta_{V}\right)$. Therefore, there is only one parameter from the modulation. In this paper, we treat $\Delta_{\phi}$ and $\Delta_{V}$ as independent quantities.

25 Symmetries I and II remain valid even if there are couplings beyond next-nearest neighbors.

${ }^{26}$ We have carried out calculations with the following sets of parameters: $\left(\Delta_{\phi}, \Delta_{V}, t_{2}\right)=(0.1 i, 0,0.1 j),(0,0.1 i, 0.1 j)$, and $(0.1 i, 0.1 i, 0.1 j)$, where $i$ and $j$ are non-negative inte- gers below 5 and 9 respectively. That is, 180 Hofstadter spectra have been generated.

27 The parameter $(2 / \pi) \beta$ in Ref. 13 is the same as our $\Delta_{\phi}$. Therefore, its Fig. 1(b) with $\beta=0.2 \pi$ is the figure with $\Delta_{\phi}=0.4$. However, the edges of subbands for a few simple fractions, e.g., $p / q=2 / 3$, in their Fig. 1(b) fail to align with nearby edges when the uniform flux is slightly varied.

28 M. C. Chang and Q. Niu, Phys. Rev. B 53, 7010 (1996).

29 Y. Hatsugai, X. G. Wen, and M. Kohmoto, Phys. Rev. B 56, 1061 (1997). 
FIG. 1: A square lattice with checkboard field modulation. The magnetic flux through the left (right) plaquette is $\phi+2 \pi \Delta_{\phi}\left(\phi-2 \pi \Delta_{\phi}\right)$. The scalar potentials at the lattice points indicated by solid and empty dots are $\Delta_{V}$ and $-\Delta_{V}$ respectively.

FIG. 2: The Hofstadter spectrum with the following parameters: $\left(\Delta_{\phi}, \Delta_{V}, t_{2}\right)=(0.1,0,0)$.
FIG. 3: The Hofstadter spectrum with the following parameters: $\left(\Delta_{\phi}, \Delta_{V}, t_{2}\right)=(0.1,0.1,0)$. 
FIG. 4: The Hofstadter spectrum with the following parameters: $\left(\Delta_{\phi}, \Delta_{V}, t_{2}\right)=(0.1,0.1,0.5)$.

FIG. 5: The Hofstadter spectrum with the following parameters: $\left(\Delta_{\phi}, \Delta_{V}, t_{2}\right)=(0.5,0.3,0.7)$.

FIG. 6: The variation of band widths as (a) $\Delta_{V}$, (b) $\Delta_{\phi}$, and (c) both $\Delta_{V}$ and $\Delta_{\phi}$ are tuned. The values of $\phi / 2 \pi=2 / 5$ and $t_{2}=0$ are fixed. 
This figure "fig1.jpg" is available in "jpg" format from: http://arxiv.org/ps/cond-mat/0308338v1 
This figure "fig2.gif" is available in "gif" format from: http://arxiv.org/ps/cond-mat/0308338v1 
This figure "fig3.gif" is available in "gif" format from: http://arxiv.org/ps/cond-mat/0308338v1 
This figure "fig4.gif" is available in "gif" format from: http://arxiv.org/ps/cond-mat/0308338v1 
This figure "fig5.gif" is available in "gif" format from: http://arxiv.org/ps/cond-mat/0308338v1 
This figure "fig6.jpg" is available in "jpg" format from: http://arxiv.org/ps/cond-mat/0308338v1 